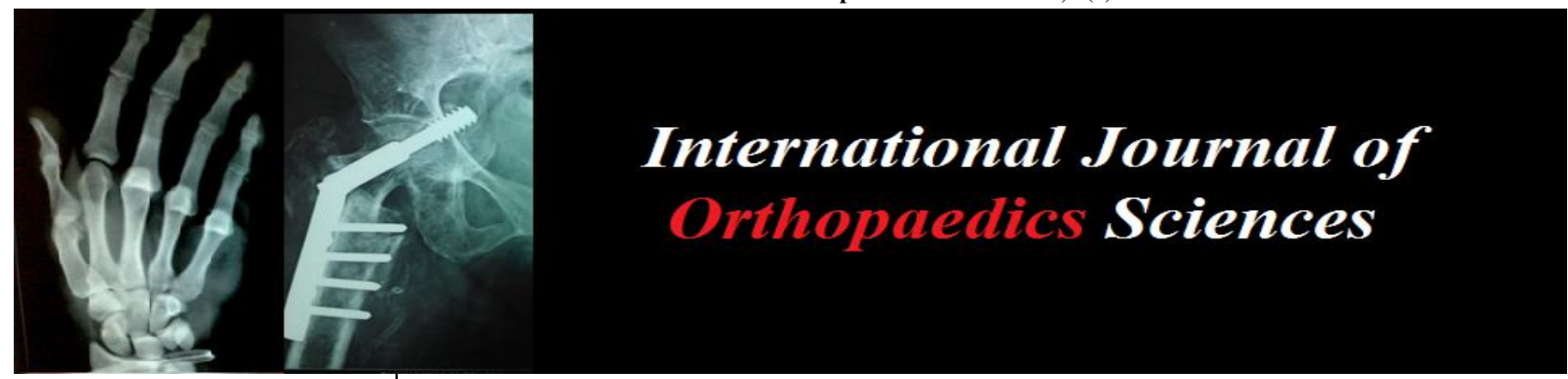

E-ISSN: 2395-1958

P-ISSN: 2706-6630

IJOS 2021; 7(4): 399-405

(C) 2021 IJOS

www.orthopaper.com

Received: 28-06-2021

Accepted: 30-07-2021

\section{Thirunthaiyan MR}

Department of Orthopaedics,

Sri Ramachandra Medical

College and Research Institute,

Chennai, Tamil Nadu, India

Mohamed N Ashik

Department of Orthopaedics,

Sri Ramachandra Medical

College and Research Institute,

Chennai, Tamil Nadu, India

Tarun Prashanth KR

Department of Orthopaedics,

Sri Ramachandra Medical

College and Research Institute,

Chennai, Tamil Nadu, India

Dorai Kumar R

Department of Orthopaedics, Sri Ramachandra Medical College and Research Institute, Chennai, Tamil Nadu, India

Corresponding Author: Mohamed N Ashik Department of Orthopaedics, Sri Ramachandra Medical College and Research Institute, Chennai, Tamil Nadu, India

\section{Effectiveness of lateral pinning in pediatric supracondylar humerus fractures}

\author{
Thirunthaiyan MR, Mohamed N Ashik, Tarun Prashanth KR and Dorai \\ Kumar R
}

DOI: https://doi.org/10.22271/ortho.2021.v7.i4f.2908

\section{Abstract}

Introduction: Supracondylar Humerus fractures remain one of the most common fractures in paediatric population, requiring operative management. Closed/open reduction and Percutaneous pinning with $\mathrm{K}$ wire, remains the mainstay of treatment for Types $2 \mathrm{~b}$ and 3 Gartland's fracture. Despite the hovering controversies, cross pinning remains the most followed technique owing to its higher stability, at the cost of slight increased chances of ulnar nerve injury and harrowing cosmetic outcome. On the other hand, lateral pinning technique provides complete safety against ulnar nerve injury. This study involves functional and radiological outcome assessment of 42 children with Gartland's Type 2 and 3 fractures who have undergone reduction and $\mathrm{k}$ wire fixation via lateral pinning.

Methodology: This is a prospective study done in SRIHER from Jan 2016-Sept 2020. All the patients with displaced supracondylar fractures with Gartland's type 2 and 3 were included. Preop, postop and intraop assessment were done with respect to Ulnar nerve palsy, Baumann's angle, Flynn criteria till 14 weeks postop.

Results: We analyzed a total of 42 pediatric populations (28 male and 14 female) who sustained Gartlands $2(71 \%)$ and $3(28 \%)$ fractures. Among the participants who underwent lateral pinning, 24 patients $(57.1 \%) 2 \mathrm{k}$ wires were used and in 18 patients $(42.9 \%) 3 \mathrm{k}$-wires were used. Among the lateral pin fixation, $24(57.1 \%)$ participants had divergent pin fixation and $18(42.9 \%)$ participants had parallel pin fixation. Flynn criteria showed good to excellent results, achieved in 34 cases and Fair results were seen in 8 cases. The difference in BA from immediate postop to 6 weeks and 10 weeks after surgery among divergent and parallel pin fixation showed no statistical significance. There was no report of iatrogenic nerve injury in any participants.

Conclusion: We suggest lateral pinning technique with the use of 3 divergent pins gives a good stable configuration in supracondylar humerus fractures, also avoiding the risk of ulnar nerve injury.

Keywords: Lateral pinning, supracondylar humerus fracture, divergent configuration

\section{Introduction}

Most common fracture encountered among the pediatric population is the supracondylar fracture of humerus ${ }^{[1]}$, among which the extension type is more common consisting of $90 \%$. It is one common fracture of the pediatric age group that needs surgical stabilization ${ }^{[2]}$. The supracondylar region of humerus is actually a thinner part of bone which is more prone for fractures. Gartland's classification is used to define the types of supracondylar humerus fractures with type 1 being a stable, undisplaced fracture which hardly needs surgical intervention. Gartland's type 2 and 3 are usually associated with high degree of angulation and displacement and are contenders for surgical fixation preceded by closed or open reduction ${ }^{[3]}$, and the technique involves reduction followed by percutaneous pinning as the mainstay treatment for such displaced fractures to prevent complications like cubitus varus deformity and non-union ${ }^{[4]}$, and it is the effective way to maintain the anatomic reduction following the closed reduction and it was first described by Casiano in $1960^{[5]}$. The percutaneous pinning is with Kirschner's wire that can be applied with various configurations to keep the fracture stabilized following the reduction. The common types of pin fixation include the lateral pinning with two or three K-wires with lateral entry only or the cross pinning with at least one pin on the medial and lateral side ${ }^{[6]}$.

There is still a debate on the preference of the configurations used for reduction, the cross 
pining is the commonly done technique in the recent past decade which includes insertion of one pin medially and one pin laterally through the epicondyles, hence the name cross pining and it expresses an enhanced mechanical stability of the configuration, but this technique has a potential disadvantage with injury to ulnar nerve and it averages from $0-15 \%$, with careful isolation of the nerve during pinning, which needs skin incision that poses cosmetic disadvantage [7]. Theoretically on the other hand, the lateral entry pins may have an equal or a slight reduced mechanical stability of configuration but the important advantage is by guaranteed avoidance of any injury to the ulnar nerve ${ }^{[8]}$. Similarly, the diameter of k-wire and the number of $\mathrm{K}$-wire is associated with the stability of the construct ${ }^{[9]}$.

Various controversies appear on the best type of reduction and fixation method and we hypothesize that lateral pinning of K-Wire has good results, in terms of functional outcomes and stability in supracondylar humerus fractures in pediatric age group. In our study, we evaluated the functional and radiological outcomes of 42 pediatric Grade 2 and 3 Gartland's displaced supracondylar humerus fractures.

\section{Methodology}

This is a non-randomized prospective study done in Sriramachandra institute of higher education and research for a period of 18 months from Jan 2016 to Sep 2020. We included the patients ageing between 3 to 13 years who had a closed isolated supracondylar fracture of humerus under Gartland type 2 with Wilkins type B fracture and Gartland type 3 fractures. We excluded all the patients with compound fractures or those with associated Claw hand deformity or Erb's palsy. Informed consent was taken from parents/ guardian and detailed history was taken. Preoperatively the radial pulse and ulnar nerve sensations were assessed and radiographs of the injured elbow was taken and then the elbow is splinted until surgery. The Fractures are classified as per Gartland's classification. Baumann's angle is calculated pre-operatively.

Anesthesia fitness is obtained for all patients, and the child undergoes the procedure under General anesthesia. All the patients' deformity was corrected by closed reduction and it was assessed with fluoroscopic assistance. The medial or lateral displacement was manipulated under direct vision and adequate reduction is confirmed by the anterior humeral line that passes center through the center of capitellum found on the true lateral view. The reduction is maintained by using 2 or more $\mathrm{K}$-wires fixed parallel or divergent pins and the size of $\mathrm{K}$-wire used is $1.6 \mathrm{~mm}$ in younger children and $2 \mathrm{~mm}$ in older children. After the procedure, above elbow slab was applied with elbow in 80-90 degrees of flexion. Broad spectrum antibiotics are given preoperatively and postoperatively for 2 shots.

\section{Post-operative assessment}

At 6 months post-op the radiological assessment is done using Baumann's angle in the antero-posterior radiograph of the elbow. Under General anesthesia, the K-Wires are removed in toto.

At 8 months post-op the functional outcomes are measured with the help of Flynn criteria and the loss of motion is measured with a Goniometer.

At 14 weeks post-op the functional outcome was done using Flynn criteria and the radiological assessment was done using Baumann's angle in the antero-posterior radiograph of elbow The normal Baumann angle is from 64-81, and the significance is that for every 5 degree change in the angle, there will be a 2 degree change in carrying angle.

The collected data were analyzed with IBM @SPSS Statistics software 23.0 version. After dividing the data collected into quantitative and qualitative variables, the data was analyzed using frequency analysis, percentage analysis, mean, S.D, Mann Whitney U test, Chi square test. Using statistical tool, the probability $(\mathrm{p})$ of $<0.05$ was considered as significant. $P<0.05$ was considered as significant and $P<0.01$ was considered as highly significant.

\section{Ethical approval}

This study was approved by Institutional Research ethics committee, Sri Ramachandra Institute of Higher education and research.

\section{Results}

We analyzed a total of 42 pediatric populations, in which the number of male children were $28(66.7 \%)$ and 14 females $(33.3 \%)$ [Tab 1 and Fig 1]. Majority of study population were in age group between 3-6 years. Mean age of the study participants who underwent lateral pin fixation was 6.1 years. Majority of the study patients who underwent lateral pinning were for Gartland's type $3(71.3 \%$ in lateral pin fixation were Gartland's type 3 ), and $28.6 \%$ in lateral pinning group had Gartland's type 2 fractures [Tab 2 and Fig 2]. All the patients underwent closed reduction and none needed open reduction. Among the participants who underwent lateral pinning, we either used 2 pins or 3 pins were in 24 patients $(57.1 \%)$ we used $2 \mathrm{k}$ wires and in 18 patients (42.9\%) $3 \mathrm{k}$-wires were used [Tab 3 and Fig 3]. Among the lateral pin fixation, 24 (57.1\%) participants had divergent pin fixation and 18 (42.9\%) participants had parallel pin fixation [Tab 4 and Fig 4]. Immediate post op means Baumann angle (BA) among the lateral pin fixation participants were 70.98 (3.73), at 6 weeks the mean and standard deviation of BA were 72.12 and 3.62 respectively. The mean and standard deviation of $\mathrm{BA}$ at 10 weeks and 14 weeks were 73.82 (3.78) and 75.13 (3.82) respectively [Tab 5]. Similarly, the mean and standard deviation of Loss of Motion (LOM) at 8 weeks, 10 weeks and 14 weeks were $30.0 \quad(5.0), 17.62 \quad(6.24), 4.52 \quad(4.4)$ respectively [Tab 6].

According to Flynn criteria, good to excellent results were achieved in 34 cases and Fair results were seen in 8 cases. The difference in BA from immediate postop to 6 weeks and 10 weeks after surgery among divergent and parallel pin fixation showed no statistical significance. But the difference in BA at 14 weeks postop showed significant difference between divergent and parallel pin fixation where mean of difference of BA of divergent pin fixation (Mean $=2.82$ ) showed least change compared to parallel pin fixation (Mean $=5.53$ ) [Fig 5]. At the end of 14 weeks change with respect to BA comparing immediate post op among 3 lateral pin and 2 lateral pin group showed 3 pin group had least change in Baumann angle of 2.75 degrees whereas 2 pin group had change of 4.9 degrees [Fig 6 and Tab 7]. This shows that using 3 lateral pins confers better stability.

There were no case of post-operative ulnar nerve palsy and infection.

Table 1: Gender distribution of study population

\begin{tabular}{|c|c|}
\hline Age & Number of patients \\
\hline Male & $28(66.7 \%)$ \\
\hline Female & $14(33.3 \%)$ \\
\hline
\end{tabular}




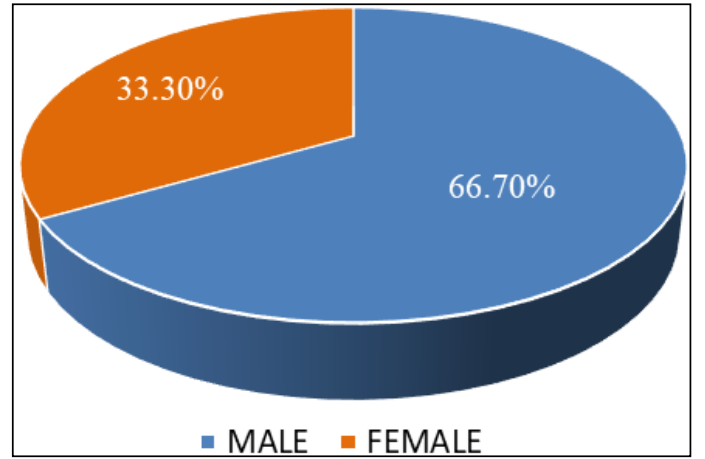

Fig 1: Gender distribution of study population

Table 2: Distribution of fracture types based on Gartland's classification

\begin{tabular}{|c|c|}
\hline Type of fracture & Lateral $\mathbf{n}(\boldsymbol{\%})$ \\
\hline Type 2B & $12(28.60 \%)$ \\
\hline Type 3 & $30(71.40 \%)$ \\
\hline Total & $42(100 \%)$ \\
\hline
\end{tabular}

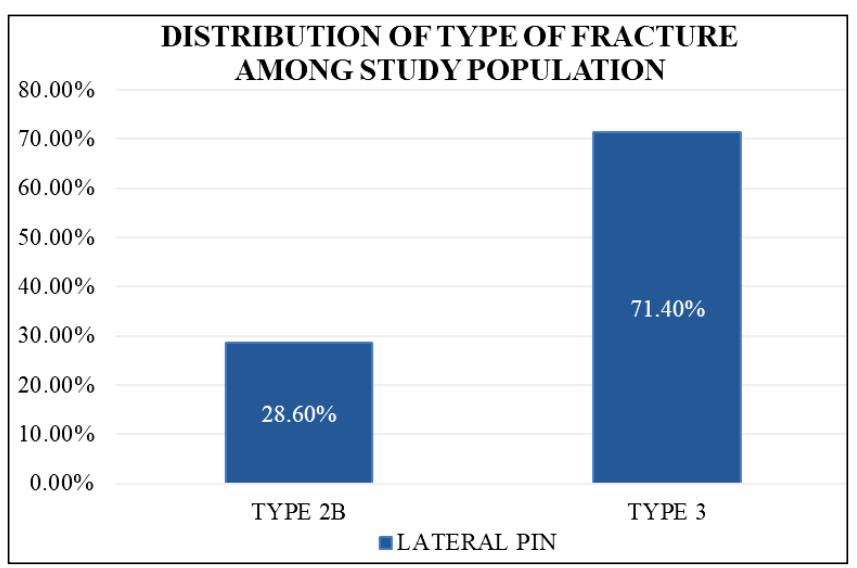

Fig 2: Distribution of type of fracture among study population

Table 3: Number of pins used among lateral pin fixation group

\begin{tabular}{|c|c|c|}
\hline No. of pins & Frequency & Percentage \\
\hline 2 & 24 & $57.1 \%$ \\
\hline 3 & 18 & $42.9 \%$ \\
\hline Total & 42 & $100 \%$ \\
\hline
\end{tabular}

Table 5: Post-operative differences in Lateral pin fixation group

\begin{tabular}{|c|c|c|c|c|c|}
\hline & Immediate Postop Mean (SD) & 6 Weeks mean (SD) & 8 Weeks mean (SD) & 10 Weeks mean (SD) & 14 Weeks mean (SD) \\
\hline BA & $70.98(3.73)$ & $72.12(3.62)$ & --- & $73.82(3.78)$ & $75.13(3.82)$ \\
\hline LOM & --- & --- & $30.0(5.0)$ & $17.62(6.24)$ & $4.52(4.4)$ \\
\hline
\end{tabular}

Table 6: Difference in Baumann's angle between divergent and parallel pin fixation

\begin{tabular}{|c|c|c|c|}
\hline Procedure & Divergent mean (SD) & Parallel mean (SD) & P-value \\
\hline Immediate post op to 6 weeks & $1.23(0.47)$ & $1.09(0.62)$ & 0.46 \\
\hline Immediate post op to 10 weeks & $2.13(1.07)$ & $3.77(2.50)$ & 0.58 \\
\hline Immediate post op to 14 weeks & $2.82(1.71)$ & $5.53(2.83)$ & 0.01 \\
\hline
\end{tabular}




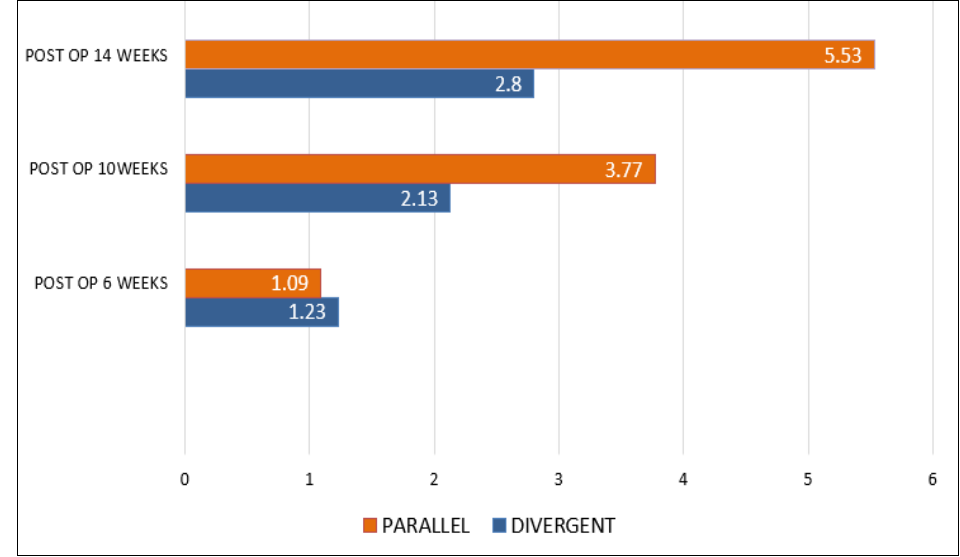

Fig 5: Difference in BA from immediate post op among pin type of lateral pin fixation

Table 7: Comparison of difference in Baumann's angle from immediate post-op up to 14 weeks among number of pins

\begin{tabular}{|c|c|c|}
\hline Number of pins & Difference in BA & P-value \\
\hline 2 & 4.90 & 0.02 \\
\hline 3 & 2.75 & \\
\hline
\end{tabular}

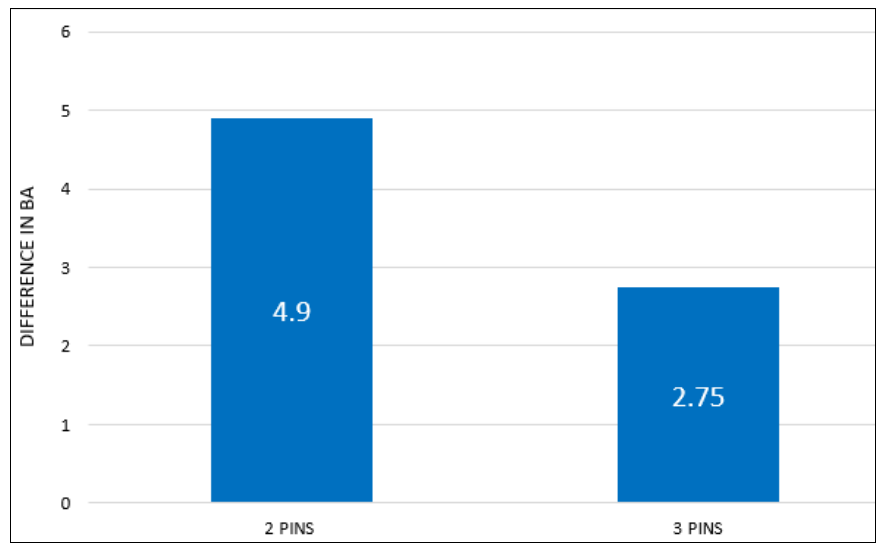

Fig 6: Difference in BA from immediate post op to 14 weeks post op among number of pins

Case example: 6 year old female with right supracondylar humerus fracture Gartland type 3 .

Procedure done: Closed reduction and Lateral pinning with 3 divergent pins.

Flynn criteria: Excellent result

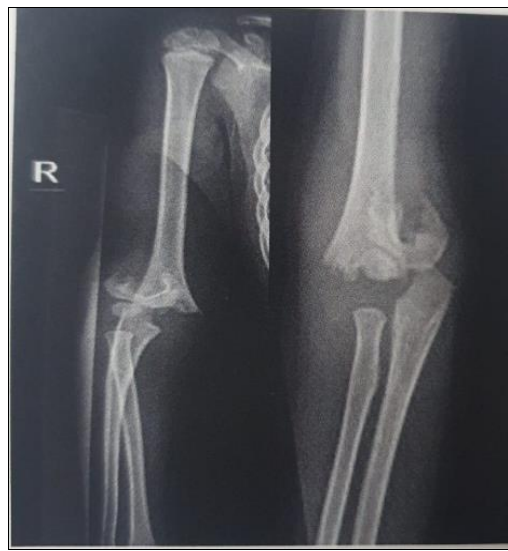

Pre-op X-ray

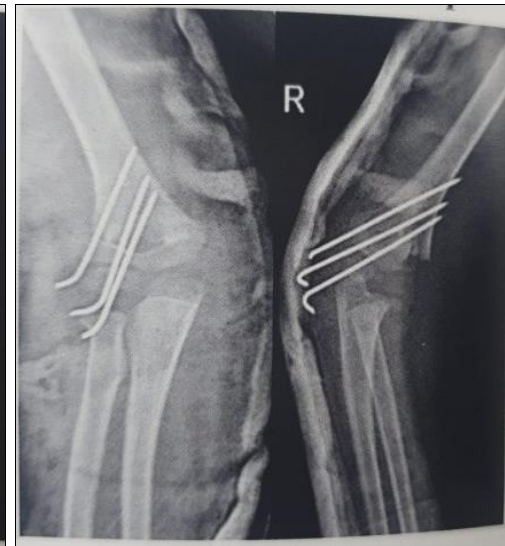

Immediate post-op 


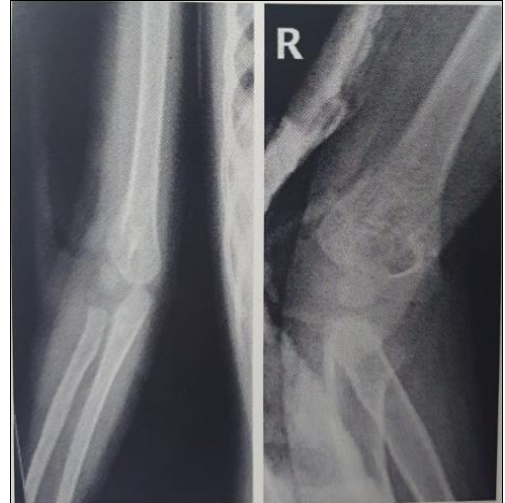

6 Weeks post-op after K-wire removal

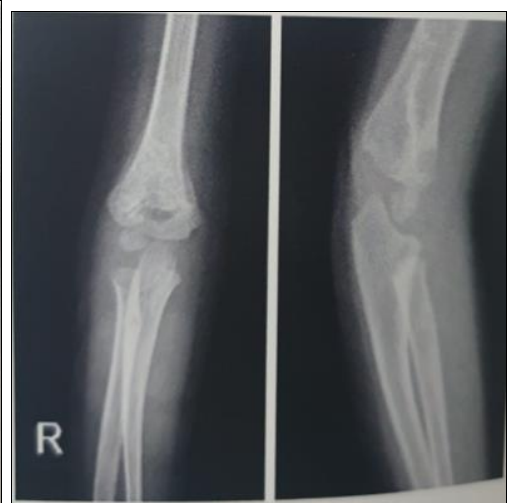

14 Weeks post-op

Case example: 4-year-old female with right supracondylar humerus fracture Gartland type 3.

Procedure done: Closed reduction and Lateral pinning with 2 pins.

Flynn criteria: Good result

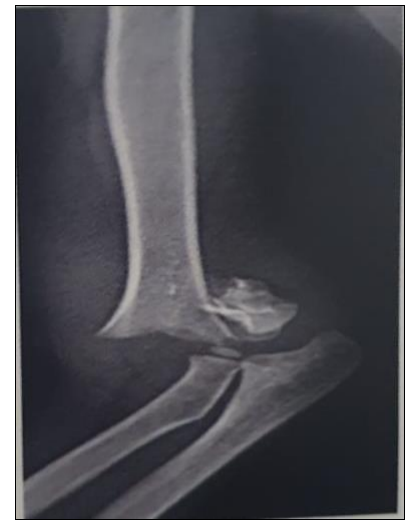

Pre-op

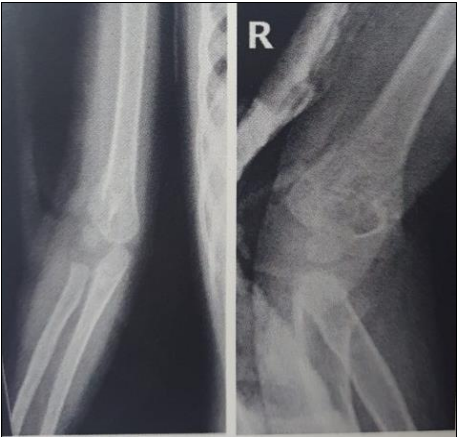

6 Weeks post-op after K-wire removal

\section{Discussion}

The major complications that can be expected during the procedure of fixation of supracondylar humerus fracture is the injury to ulnar nerve and loss of the reduction following fixation $^{[1]}$ as the treatment of choice for displaced Gartland's 2 and 3 fracture is closed or open reduction and pinning. Though the type of pinning either lateral or cross pinning of $\mathrm{K}$-wire is surgeon preference, more orthopedic professionals choose the lateral pinning to cross pinning to avoid the iatrogenic nerve injury as mentioned above ${ }^{[1]}$. Theoretically cross pinning gives better construct, but many literatures have shown the risks of loss in reduction or developing a cubitus varus deformity is same for both pinning groups.

Many analysis results disclosed that iatrogenic ulnar nerve injury mainly occurred in the crossed entry pin group ${ }^{[11]}$. Brauer et al. ${ }^{[10]}$ in his study has shown that the probability of

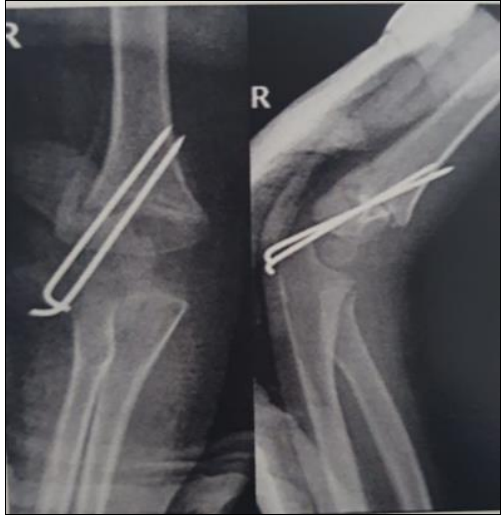

Immediate post-op

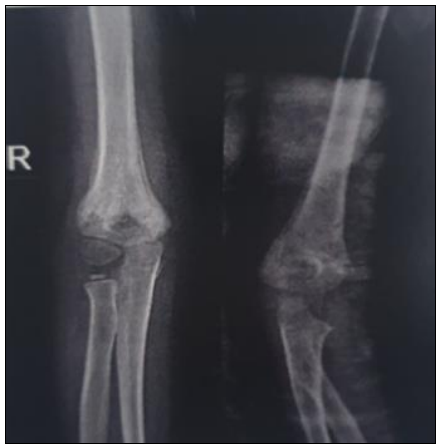

14 Weeks post-op

iatrogenic ulnar nerve injury with cross entry pins is 5 times higher than that with lateral entry pins in their systematic review study. Woratanarat et al. in his study suggested that lateral entry technique is superior to crossed pinning technique for Supracondylar humerus fracture fixation as a result of decreased risk of iatrogenic ulnar nerve injury ${ }^{[12]}$. When there is an evident expected iatrogenic nerve injury that is of a certainty in crossed pinning, its use can be minimized for its slight or same level of fixation compared to lateral pinning, making lateral k-wires supreme to cross pinning. Zhao et al. ${ }^{[4]}$ analyzed ulnar nerve injury by reviewing seven randomized control trials and added to the fact that nerve injury was higher with medial/lateral crossed entry pins than with lateral entry pins ( 3.3 times). In our study, for 24 patients 3 lateral entry pins were used and for 18 patients only 2 lateral $\mathrm{k}$-wires were used. To point to stress here is It has been 
shown that crossed pins do provide more torsional stability than do 2 lateral pins but do not offer significantly more torsional stability than do 3 lateral pins ${ }^{[14]}$, proving the efficacy of using 3 lateral entry pins instead of 2 . Lee et al. ${ }^{[13]}$ have studied the efficacy of using divergent pins in the management of displaced supracondylar fractures. In their study, $61 \%$ of the participants had sustained type 3 Gartland fracture and $39 \%$ had sustained type 2 Gartland fracture. They have shown no patients had loss of reduction with the usage of divergent pins. In our study also, majority of participants $70.3 \%$ had sustained type 3 Gartland fracture and only $29.07 \%$ of patients sustained type 2B Gartland fracture.

4.3 Also in our study, on comparing divergent and parallel lateral pinning techniques, divergent pins provided the better stability with least change of mean Baumann's angle of 2.82 degree at the end of 14 weeks whereas change in mean Baumann' s angle in parallel pins were 5.53 degree at the end of 14 weeks when compared to immediate postop. The divergent lateral pins and crossed pins had indistinguishable performances by valgus testing, and both were found to be further stable than lateral parallel pins. Lee in his study also found that 2 divergent lateral-entry pins provide greater stability in both varus and valgus loading than 2 parallel lateral-entry pins. When wires are closely positioned, the mechanical construct obtained becomes almost comparable to a single wire construct, and thus allows the distal fragment to rotate around the axis of these $\mathrm{k}$ - wires. In contrast to the divergent pins, which are inserted to a greater distance between the pins at the fracture site and the greater divergence angle between the pins to engage both medial and lateral columns. All these efforts are described for the use of 2 pins, and when 3 pins are used, no significant difference between radiological mean Baumann angles and mean lateral humerocapitellar angles for the 3 divergent pin and 3 parallel pin configurations. Gordon et al. [15] have concluded significant rotational instability on using only 2 lateral entry pins in Gartland type 3 fractures when compared with usage of 3 lateral pins which was more stable. In our study also, using 3 lateral pins confers better stability than using only 2 lateral pins.

Several studies have concluded that lateral pinning configuration confers equal stability as cross pin configuration ${ }^{[16,17]}$. Maity et al. in their study comparing the two fixation modalities cross pinning and lateral pinning in the treatment of displaced supracondylar humerus fractures have demonstrated statistically insignificant difference in outcome with respect to Flynn criteria in both the groups ${ }^{[18]}$. All the patients were addressed for range of motion during follow-up periods and were encouraged to do range of motion exercises and that improved the Loss of motion of elbow at the end of 14 weeks.

There are very few limitations of our study was that the true postoperative antero-posterior and lateral radiographs were not able to be obtained in some children since they were not cooperative that may affect the accuracy of variables and carrying angle cannot be measured in case of existing fixed flexion deformity.

\section{Conclusion}

From our study we conclude that the lateral pinning technique provides supreme stability in the treatment of displaced supracondylar fracture of humerus in pediatric population especially the use of 3 divergent lateral entry pinning with no risk of iatrogenic nerve damage or without the need of incision over the medial aspect hence producing a stable

configuration as well of cosmetic superiority.

\section{Abbrevations \\ 1 BA-Baumann's angle}

Acknowledgement

I would like to acknowledge my colleagues Dr. Kaustav Mukherjee and Dr. Nithin Venkat for their constant support.

\section{Conflict of interest: Nil.}

Fundings: Nil

\section{References}

1. Na Y, Bai R, Zhao Z et al. Comparison of lateral entry with crossed entry pinning for pediatric supracondylar humeral fractures: A meta-analysis. J Orthop Surg Res 2018;13:68.

2. Lamdan R, Liebergall M, Gefen A, Symanovsky N, Peleg E. Pediatric supracondylar humerus fractures: Effect of bone-implant interface conditions on fracture stability. J Child Orthop 2013;7(6):565-569. Doi: 10.1007/s11832013-0533-4

3. Gartland JJ. Management of supracondylar fractures of the humerus in children. Surg Gynecol Obstet 1959;109:14554.

4. Zhao JG, Wang J, Zhang P. Is lateral pin fixation for displaced supracondylar fractures of the humerus better than crossed pins in children? Clin Orthop Relat Res. 2013;471(9):2942-2953. Doi: 10.1007/s11999-013-3025-4

5. Casiano E. Reduction and fixation by pinning "banderillero" style-fractures of the humerus at the elbow in children. Mil Med 1960;125:262-264.

6. Otsuka NY, Kasser JR. Supracondylar fractures of the humerus in children. Am Acad Orthop Surg 1997;5:19-26.

7. Lyons JP, Ashley E, Hoffer MM. Ulnar nerve palsies after percutaneous crosspinning of supracondylar fractures in children's elbows. J Pediatr Orthop 1998;18:43-5.

8. Lee SS, Mahar AT, Miesen D, Newton PO. Displaced pediatric supracondylar humerus fractures: biomechanical analysis of percutaneous pinning techniques. J Pediatr Orthop 2002;22:440-3.

9. Srikumaran U, Tan EW, Erkula G, Leet AI, Ain MC, Sponseller PD. Pin size influences sagittal alignment in percutaneously pinned pediatric supracondylar humerus fractures. Journal of Pediatric Orthopaedics 2010;30(8):792-798.

10. Brauer CA, Lee BM, Bae DS, Waters PM, Kocher MS. A systematic review of medial and lateral entry pinning versus lateral entry pinning for supracondylar fractures of the humerus. Journal of Pediatric Orthopaedics 2007;27(2):181-186.

11. Barr LV. Paediatric supracondylar humeral fractures: epidemiology, mechanisms and incidence during school holidays. J Child Orthop 2014;8(2):167-170. Doi: 10.1007/s11832-014-0577-0.

12. Woratanarat, Patarawan MD, Ph.D. Angsanuntsukh, Chanika MD, Rattanasiri, Sasivimol, PhD Attia et al. Meta-Analysis of Pinning in Supracondylar Fracture of the Humerus in Children, Journal of Orthopaedic Trauma 2012;26(1):48-53. 
13. Lee YH, Lee SK, Kim BS, Chung MS, Baek GH, Gong HS et al. Three lateral divergent or parallel pin fixations for the treatment of displaced supracondylar humerus fractures in children. J Pediatr Orthop. 2008; 28(4):41722.

14. Zionts LE, McKellop HA, Hathaway R. Torsional strength of pin configurations used to fix supracondylar fractures of the humerus in children. J Bone Joint Surg Am 1994;76:253Y256.

15. Gordon JE, Patton CM, Luhmann SJ, Bassett GS, Schoenecker PL. Fracture stability after pinning of displaced supracondylar distal humerus fractures in children. J Pediatr Orthop. 2001; 21(3):313-8.

16. Foead A, Penafort R, Saw A, Sengupta S. Comparison of two methods of percutaneous pin fixation in displaced supracondylar fractures of the humerus in children. J Orthop Surg (Hong Kong) 2004;12(1):76-82.

17. Prashant K, Lakhotia D, Bhattacharyya TD, Mahanta AK, Ravoof A. A comparative study of two percutaneous pinning techniques (lateral vs medial-lateral) for Gartland type III pediatric supracondylar fracture of the humerus. J Orthop Traumatol 2016;17(3):223-9.

18. Maity A, Saha D, Roy DS. A prospective randomised, controlled clinical trial comparing medial and lateral entry pinning with lateral entry pinning for percutaneous fixation of displaced extension type supracondylar fractures of the humerus in children. J Orthop Surg Res 2012;7:6. 